\title{
Prevalence of Hepatitis B Virus Infection in Kaifeng, China: A 5-year Observation
}

\author{
Jinli Zhang ${ }^{1 \dagger}$, Chunyan $\mathrm{Ma}^{1 \dagger}{ }^{1}$, Hang Li ${ }^{1}$, Michael Steele ${ }^{2}$, and Adi Idris ${ }^{1,3 *}$ \\ ${ }^{1}$ Department of Clinical Laboratory, Kaifeng Central Hospital, Kaifeng, Henan, China \\ ${ }^{2}$ Faculty of Health Sciences, Australian Catholic University, Queensland, Australia \\ ${ }^{3}$ Menzies Health Institute Queensland, School of Medical Science, Griffith University, Gold Coast, Queensland, Australia
}

Received: June 22, 2018 / Revised: July 14, 2018 / Accepted: July 17, 2018

\begin{abstract}
Hepatitis B is a major health problem in China. However, little is known about the prevalence of hepatitis $B$ virus (HBV) in Kaifeng, the major urban capital of Henan province, China. We found that HBV prevalence increased with age and that chronic HBV was predominant in adult males in Kaifeng. The HBV prevalence remained unchanged over a 5 -year period for all age groups. Alarmingly, 25\% of the population remained unvaccinated and potentially susceptible to future HBV infection. HBV immunization and health education initiatives should be carried out in this population to further reduce the overall prevalence of HBV.
\end{abstract}

Keywords: Hepatitis B virus, Kaifeng, China, HBsAg

Hepatitis B virus (HBV) infection is the main risk factor for hepatocellular carcinoma. Hepatitis $B$ vaccination is the most effective measure to prevent HBV infection. For those who are already infected with HBV, progression to chronic liver disease can occur and be treated with antiviral agents. However, this contributes to a significant healthcare-associated cost to the economy. HBV is endemic in China. The prevalence of HBV infections in China remains high [1] despite the introduction of the National Expanded Programme on Immunization. Furthermore, there is a stark difference in HBV infection rates among different regions in China [2]. Although the HBV sero-prevalence in Henan province have been reported [3], little is known about the prevalence of $\mathrm{HBV}$ in Kaifeng, the major urban capital of Henan province. A general population-based study on the prevalence of HBV was therefore conducted in Kaifeng between 2013-

\section{*Corresponding author}

Tel: +617555527709, Fax: +617555527709

E-mail: a.idris@griffith.edu.au

${ }^{\dagger}$ These authors contributed equally to this work.

(c) 2018, The Korean Society for Microbiology and Biotechnology
2017.

This retrospective survey was conducted at Kaifeng Central Hospital, Kaifeng, China based on data from January 2013 and December 2017. Approval by the Hospital Ethics Committee waived the need for informed consent. Kaifeng Central Hospital is designated as a health care centre by the Kaifeng City government. The total number of subjects that visited the hospital between 2013 and 2017 was over 220,000. However, this study only included samples from subjects who visited the hospital for general health routine check-ups. Venous blood serum from 52,848 subjects (27,400 men and 25,448 women from age 10 to 97 years old) were obtained and subjected to enzyme-linked immunosorbent assay (ELISA) for HBV surface antigen (HBsAg) (Kehua HBsAg kit, Cut-off value $\geq 0.1$ x mean absorbance of negative control (N), Shanghai Kehua Biotech, Shanghai, China) and antibodies against HBV surface antigen (anti-HBs) and total HBV core antigen (antiHBc) (Kehua Anti-HBs and Anti-HBc kits, Cut-off value $\geq 2.1 \times$ N, Shanghai Kehua Biotech, China) by aid of the Freedom EVO-2 Clinical 150 liquid handler (Tecan, 
Table 1. Pooled prevalence of HBV infection and vaccine-induced immunity of Kaifeng Central Hospital patients throughout study period (2013-2017).

\begin{tabular}{|c|c|c|c|c|c|}
\hline & $\begin{array}{l}\text { All patients } \\
(n=52848)\end{array}$ & $\begin{array}{l}\text { Past and present } \\
\text { infection }(n=7838)\end{array}$ & $\begin{array}{l}\text { Chronic infection } \\
\qquad(n=2443)\end{array}$ & $\begin{array}{c}\text { Vaccine-induced } \\
\text { immunity }(n=31228)\end{array}$ & $\begin{array}{l}\text { Susceptible } \\
(n=13431)\end{array}$ \\
\hline \multicolumn{6}{|l|}{ Age } \\
\hline Mean (SD) & 49.57 (15.09) & $50.42(14.54)$ & $47.50(14.02)$ & 48.45 (15.19) & $51.73(14.93)$ \\
\hline Median (IQR) & $50(21)$ & $50(20)$ & $48(21)$ & $48(22)$ & $52(20)$ \\
\hline Min, Max & 10,97 & 10,97 & 10,97 & 10,97 & 10,96 \\
\hline 10-19 Years N(\%) & $672(1.3)$ & $35(0.4)$ & $23(0.9)$ & 459 (1.5) & $177(1.3)$ \\
\hline 20-29 Years N(\%) & $5089(9.6)$ & $584(7.5)$ & $247(10.1)$ & $3386(10.8)$ & $1079(8.0)$ \\
\hline 30-39 Years N(\%) & $8255(15.6)$ & 1300 (16.6) & $436(17.8)$ & $5412(17.3)$ & $1476(11.0)$ \\
\hline 40-49 Years N(\%) & $11824(22.4)$ & $1814(23.1)$ & $633(25.9)$ & 7098 (22.7) & $2824(21.0)$ \\
\hline 50-59 Years N(\%) & $12736(24.1)$ & $1923(24.5)$ & $605(24.8)$ & 7076 (22.7) & 3651 (27.2) \\
\hline 60-69 Years N(\%) & $8876(16.8)$ & $1354(17.3)$ & $353(14.4)$ & 4886 (15.6) & 2587 (19.3) \\
\hline 70-79 Years N(\%) & $4323(8.2)$ & $651(8.3)$ & $112(4.6)$ & $2365(7.6)$ & $1290(9.6)$ \\
\hline 80-89 Years N(\%) & $1016(1.9)$ & $170(2.2)$ & $31(1.3)$ & $516(1.7)$ & $327(2.4)$ \\
\hline $90+$ Years N(\%) & $57(0.1)$ & $7(0.1)$ & $3(0.1)$ & $30(0.1)$ & $20(0.1)$ \\
\hline \multicolumn{6}{|l|}{ Gender } \\
\hline Male, N (\%) & $27400(51.8)$ & $4206(53.7)$ & $1479(60.5)$ & $16210(51.9)$ & $6815(50.7)$ \\
\hline Female, N (\%) & $25448(48.2)$ & $3632(46.3)$ & 964 (39.5) & $15018(48.1)$ & $6616(49.3)$ \\
\hline Ratio M:F & 1.077 & 1.158 & 1.534 & 1.079 & 1.030 \\
\hline Prevalence All \%(Cl) & - & $14.8(14.5,15.1)$ & $4.6(4.4,4.8)$ & $59.1(58.7,59.5)$ & $25.4(25.0,25.8)$ \\
\hline 10-19 Years \%(Cl) & - & $5.2(3.8,7.2)$ & $3.4(2.3,5.1)$ & $68.3(64.7,71.7)$ & $26.3(23.2,29.8)$ \\
\hline 20-29 Years \%(Cl) & - & $11.5(10.6,12.4)$ & $4.9(4.3,5.5)$ & $66.5(65.2,67.8)$ & $21.2(20.1,22.3)$ \\
\hline 30-39 Years \%(Cl) & - & $15.8(15.0,16.6)$ & $5.3(4.8,5.8)$ & $65.6(64.5,66.6)$ & $17.9(17.1,18.7)$ \\
\hline 40-49 Years \%(Cl) & - & $15.3(14.7,16.0)$ & $5.4(5.0,5.8)$ & $60.0(59.1,60.9)$ & $23.9(23.1,24.7)$ \\
\hline $50-59$ Years \%(Cl) & - & $15.1(14.5,15.7)$ & $4.8(4.4,5.1)$ & $55.6(54.7,56.4)$ & $28.7(27.9,29.5)$ \\
\hline 60-69 Years \%(Cl) & - & $15.3(14.5,16.0)$ & $4.0(3.4,4.4)$ & $55.1(54.0,56.1)$ & $29.2(28.2,30.1)$ \\
\hline 70-79 Years \%(Cl) & - & $15.1(14.0,16.2)$ & $2.6(2.2,3.1)$ & $54.7(53.2,56.2)$ & $29.8(28.5,31.2)$ \\
\hline $80-89$ Years \%(Cl) & - & $16.7(14.6,19.2)$ & $3.1(2.2,4.3)$ & $50.8(47.7,53.9)$ & $32.2(29.4,35.1)$ \\
\hline $90+$ Years \%(Cl) & - & $12.3(6.1,23.3)$ & $5.3(1.8,14.4)$ & $52.6(39.9,65.0)$ & $35.1(24.0,48.1)$ \\
\hline
\end{tabular}

Footnote: ${ }^{\mathrm{N}}(\%) ;{ }^{\mathrm{b}} \mathrm{Cl}(\%)$.

Switzerland) and absorbance read on an Infinite F50 microplate reader (Tecan, Switzerland). If an initial weak reaction was observed for any of the markers in the first ELISA test, a repeat test was performed. Deidentified data were entered into IBM SPSS Statistics 22.0 (SPSS Institute, USA). Before analysis, all variables were reviewed for accuracy of data entry and missing values. Due to the large sample size involved, the statistical analysis is focused primarily on frequencies and percentages.

The prevalence for cases positive for the three markers and a combination of them are summarized in Table 1 by sex and decade of years of age. The average age of tested patients was 49.57 years old (standard deviation $(\mathrm{SD})=15.09)$ (Table 1). Past or present HBV infection was defined as the presence of anti-HBc. If anti-HBc positive subjects were tested positive for HBsAg, they are regarded as chronically infected with HBV. On the other hand, those who were anti-HBs positive and negative for anti-HBc were considered vaccine-immune. The overall prevalence (all ages and both sexes combined) of past and present infection during the 2013-2017 period was $14.8 \%$ (95\% confidence interval [CI], 14.5\%-15.1\%) (Table 1). The prevalence of HBV infection increases 
A

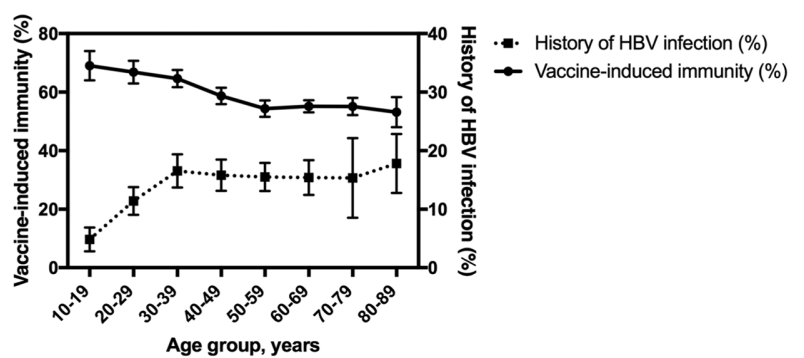

C

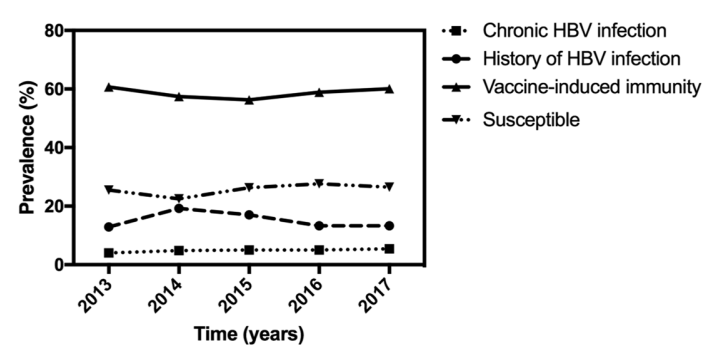

B

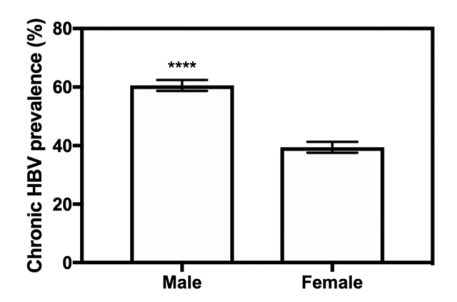

Fig. 1. (A) Crude prevalence of markers for HBV infection and vaccine-Induced immunity by age (>90 years old group was excluded due to low sample number), 2013-2017. Data is representative of mean \pm standard deviation (SD). (B) Prevalence of markers for chronic infection by sex, 2013-2017. (C) Prevalence of HBV infection, vaccine-induced immunity and susceptible groups by time.

with age, consistently maintained in the 30-39 years old age group onwards (Fig. 1A). On the other hand, the overall prevalence of markers of vaccine-induced immunity was 59.1\% (95\% CI, 58.7\%-59.5\%). Prevalence of vaccine-induced immunity slightly decreased with age, ranging from $68.3 \%$ (95\% CI, 64.7\%-71.7\%) among persons aged $10-19$ years to $50.8 \%$ (95\% CI, $47.7 \%-53.9 \%)$ among persons 80-89 years of age (Table 1 and Fig. 1A). The prevalence of patients with chronic HBV infection was $4.6 \%$, which represents 2,443 infected persons, especially in the 40-49 and 50-59 age groups (Table 1). Notably, there was a significantly higher frequency of patients with chronic HBV in males than in females (Fig. 1B). Alarmingly, $25.4 \%(n=13,431)$ of tested patients were negative for all markers (uniform across all age groups) indicative of not yet being vaccinated and are therefore susceptible to HBV infection (Table 1). Among the twenty individuals aged 90 years old and older who were tested, $35.1 \%$ (95\% CI, $24 \%-48.1 \%)$ were susceptible to $\mathrm{HBV}$ infection; the representativeness of this estimate is uncertain due to the low sample numbers in this group. There was no notable difference in the prevalence of HBV infection, vaccine-induced immunity and susceptible groups over time (Fig. 1C).

A recent national HBV blood screening survey revealed a $0.07 \% \mathrm{HBV}$ prevalence (both acute and chronic HBV) in Kaifeng [4]. Importantly, they reported that HBV prevalence in Kaifeng was the lowest compared to other tested regions in China. In contrast, we found a high prevalence rate for chronic HBV (4.6\%) over the 5 year-period. It is important to note that the study by Ling et al., [4] employed a more stringent testing algorithm, using two independent $\mathrm{HBsAg}$ detection kits of different brands and final confirmation by a neutralization assay. Another possibility to explain such low HBV prevalence in that study was the restrictive and stringent blood donor screening process employed compared to our study, which did not apply any strict exclusion criteria. Nonetheless, our prevalence figures resemble closely to HBV prevalence reported previously in Henan province [3].

The large burden of chronic HBV infection in adult males (between 30-49 years old) highlights the need to improve screening programs to identify chronic infected persons, most whom remain asymptomatic until the late stages of liver disease. The large prevalence in males have been seen previously in the South-eastern $[5,6]$ and central [3] regions of China. The high prevalence of those with a past and present HBV infection in the older age groups ( $>40$ years old) mirrors the low percentage 
vaccine-induced immunity seen in those age groups. We report no significant change in HBV prevalence (both past and present and chronic HBV) or vaccine-immunity over time suggesting that little intervention has been done to address this issue over the 5 -year period. The presence of an acute infection could not be confirmed here as immunoglobulin (Ig) M antibodies to hepatitis core antigen (IgM anti-HBc) were not measured in patient blood. Although we established chronic HBV infection in some patients, we did not further verify the presence of HBV DNA in this study. We cannot rule out the possibility of occult HBV infections [7] in some patients whose serology results present as HBsAg negative but anti-HBc positive. In such cases, a HBV DNA detection is therefore crucial to confirm this.

In summary, our 5-year study highlights that HBV prevalence increases with age and that chronic HBV is predominant in adult males in Kaifeng. Alarmingly, we report that the prevalence of chronic HBV in Kaifeng to be higher than the current national average [8]. Furthermore, HBV prevalence remains unchanged over the 5-year period for all age groups highlighting that HBV infection remains a public health problem in Kaifeng. It is clear that early neonate HBV testing together with adult vaccination are two critical factors for controlling HBV endemicity in China [9]. Awareness programmes and second line treatment implementation could minimize the prevalence and mortality of HBV significantly. There still remains over a quarter of the patients (in nearly all age groups) tested in our study who are susceptible to a future HBV infection as they have not yet been vaccinated. Future active HBV vaccination community programmes are needed to address this issue. Our findings provide new information for tackling HBV in Kaifeng. Providing health education programmes to the public on HBV should be given more attention on local government health policy.

\section{Acknowledgments}

No funding to acknowledge. We would like to acknowledge the technical staff at the Department of Clinical Laboratory, Kaifeng Central Hospital for assisting us in patient sample collection and record keeping.

\section{Conflict of Interest}

The authors have no financial conflicts of interest to declare.

\section{References}

1. Li W, Gao Z, Yang C, Li J, Li L, Lv R, et al. 2013. The estimation of prevalence, incidence, and residual risk of transfusion-transmitted human hepatitis $B$ infection from blood donated at the Anhui blood center, China, from 2009 to 2011. PLoS One 8: e73472.

2. Chen YS, Li L, Cui FQ, Xing WG, Wang L, Jia ZY, et al. 2011. [A seroepidemiological study on hepatitis $C$ in China]. Zhonghua Liu Xing Bing Xue Za Zhi. 32: 888-891.

3. Yong Hao G, Da Xing F, Jin X, Xiu Hong F, Pu Mei D, Jun L, et al. 2017. The prevalence of hepatitis $B$ infection in central China: An adult population-based serological survey of a large sample size. J. Med. Virol. 89: 450-457.

4. Li L, Han T, Zang L, Niu L, Cheng W, Lin H, et al. 2017. The current incidence, prevalence, and residual risk of hepatitis $B$ viral infections among voluntary blood donors in China. BMC Infect Dis. 17: 754.

5. Chen P, Xie Q, Chen T, Wu J, Wu J, Ruan B, et al. 2017. Hepatitis B virus infection in hilly/mountainous regions of southeastern China: a locality-dependent epidemiology. BMC Infect Dis. 17: 809.

6. Luo Z, Xie Y, Deng M, Zhou X, Ruan B. 2011. Prevalence of hepatitis $B$ in the southeast of China: a population-based study with a large sample size. Eur. J.Gastroenterol. Hepatol. 23: 695-700.

7. Makvandi M. 2016. Update on occult hepatitis B virus infection. World J. Gastroenterol. 22: 8720-8734.

8. Zu J, Zhuang G, Liang P, Cui F, Wang F, Zheng H, et al. 2017. Estimating age-related incidence of $\mathrm{HBsAg}$ seroclearance in chronic hepatitis $B$ virus infections of China by using a dynamic compartmental model. Sci Rep. 7: 2912.

9. Goyal A, Murray JM. 2017. Roadmap to control HBV and HDV epidemics in China. J. Theor. Biol. 423: 41-52. 